\title{
MicroRNA-497 inhibits the proliferation, migration and invasion of human bladder transitional cell carcinoma cells by targeting E2F3
}

\author{
YIXIAO ZHANG ${ }^{1,2}$, ZHE ZHANG $^{1}$, ZHENHUA LI $^{1}$, DAXIN GONG $^{1}$, BO ZHAN ${ }^{1}$, \\ XIAOJUN MAN ${ }^{1}$ and CHUIZE KONG ${ }^{1}$
}

\begin{abstract}
${ }^{1}$ Department of Urology Surgery, The First Affiliated Hospital of China Medical University, Shenyang, Liaoning 110001;
${ }^{2}$ Department of Urology Surgery, Shengjing Hospital of China Medical University, Shenyang, Liaoning 110004, P.R. China
\end{abstract}

Received January 17, 2016; Accepted February 14, 2016

DOI: 10.3892/or.2016.4923

\begin{abstract}
Accumulating evidence indicates that microRNAs (miRNAs) play critical roles in regulating cellular processes, such as cell growth and apoptosis, as well as cancer progression and metastasis. Low expression of miR-497 has been observed in breast, colorectal and cervical cancers. Human bladder transitional cell carcinoma (BTCC) progression typically follows a complex cascade from primary malignancy to distant metastasis, but whether the aberrant expression of miR-497 in BTCC is associated with malignancy, metastasis or prognosis remains unknown. In the present study, we found that miR-497 was markedly downregulated in BTCC tissue samples when compared with that noted in adjacent normal tissues, and low expression of miR-497 was correlated with poor prognosis in BTCC patients. We also found that overexpression of miR-497 inhibited the proliferation, migration and invasion of bladder cancer cells by downregulating E2F3 (an miR-497 target gene) mRNA and protein and that siRNA against E2F3 inhibited cell proliferation, migration and invasion, which was similar to the effect of miR-497 overexpression in the BTCC cells. Our experimental data indicated that miR-497 mediates the in vitro proliferation, migration and invasion of BTCC cells. Together, these results suggest that miR-497 may represent a novel prognostic indicator, a biomarker for the early detection of metastasis and a target for gene therapy of BTCC.
\end{abstract}

Correspondence to: Dr Chuize Kong, Department of Urology Surgery, The First Affiliated Hospital of China Medical University, 155 Nanjing Street, Heping, Shenyang, Liaoning 110001, P.R. China E-mail: kongchuize_cmu@sina.cn

Abbreviations: BTCC, bladder transitional cell carcinoma; miRNAs, microRNAs; miR-497, microRNA-497

Key words: human bladder transitional cell carcinoma, miR-497, cancer progression, E2F3

\section{Introduction}

Human bladder transitional cell carcinoma (BTCC) is one of the most frequent malignant tumors and its incidence is increasing worldwide (1). Despite many advances in cancer diagnosis and treatment in recent decades, the outcome of patients with advanced BTCC remains poor. There is, therefore, a tremendous and urgent need for elucidating the underlying molecular mechanisms of BTCC tumorigenesis and for developing novel therapeutic strategies, including treatments aimed at specific molecular targets.

MicroRNAs (miRNAs), which are short, highly conserved non-coding RNAs, regulate the expression of messenger RNAs (mRNAs) by binding to their 3'-untranslated region (3'-UTR) $(2,3)$. miRNAs play a critical role in processes related to cancer by acting as oncogenes or tumor suppressors in the regulation of carcinogenesis, metastatic capacity and drug resistance. Low expression of miR-497 has been observed in breast, colorectal and cervical cancers (4). Recent studies have shown that miR-497 suppresses angiogenesis and invasion of ovarian cancer by targeting vascular endothelial growth factor A (5). Li et al reported that miRNA-497 modulates gastric cancer cell proliferation and invasion by repressing eIF4E (6). Notably, a recent study by Itesako et al found that miR-497 significantly inhibited cancer cell proliferation, migration and invasion in two bladder cell lines by targeting BIRC5 and WNT7A (7). However, the relationship between miR-497 and the prognosis of BTCC patients, and whether miR-497 targets other target genes to regulate BTCC cell growth and invasion are undefined.

Transcription factor E2F3, a key regulator of G1/S phase transition, plays major roles in regulating cell cycle progression $(8,9)$. Previous studies have shown that E2F3 overexpression is critical for the growth and survival of bladder cancer cells, and is generally correlated with poor outcomes $(3,10)$. Furthermore, BTCC patients with higher levels of E2F3 also show rates of higher mortality compared with patients with lower levels of E2F3 (3,11), suggesting that E2F3 is important for BTCC development. To date, several miRNAs, such as miR-34a, miR-20a, miR-125b and miR-217, have been confirmed to suppress cell growth and survival by targeting E2F3 in tumor cells (3,12-16). 
However, the relationship between miR-497 and E2F3 in BTCC is poorly understood.

In the present study, we found that miR-497 was significantly downregulated in BTCC cell lines and tissues. Furthermore, we identified E2F3 as a target gene of miR-497 and showed that upregulation of miR-497 inhibited cell proliferation and invasion by directly downregulating E2F3 expression in T24 and UM-UC-3 cells in vitro. Our data indicated that miR-497 functions as a tumor suppressor in BTCC development and serves as a prognostic marker for BTCC.

\section{Materials and methods}

Cell lines and culture. Human BTCC cell lines (T24, 5637, BIU-87 and UM-UC-3) and a human bladder urothelium cell line (SV-HUC-1) were purchased from the Chinese Science Institute (Shanghai, China). All cell lines were maintained in high glucose Dulbecco's modified Eagle's medium (DMEM) supplemented with $10 \%$ fetal bovine serum (FBS) (both from Gibco, Gaithersburg, MD, USA). Cells were incubated at $37^{\circ} \mathrm{C}$ in a humidified atmosphere with $5 \% \mathrm{CO}_{2}$.

Tissue specimens. Eighty paired tissue samples from BTCCs and the corresponding adjacent normal tissues were collected from the Department of Pathology of Shengjing Hospital. All tissue specimens were confirmed by pathology. The collection and use of tissues followed procedures that were in accordance with the ethical standards formulated in the Declaration of Helsinki. Patients provided informed consent prior to tissue collection. The present study was approved by the Institutional Research Ethics Committee of China Medical University.

Transfection of miRNA mimics and small interfering RNAs. An miR-497 mimic (referred to as miR-497) and negative control (NC) duplex were used for further gain-of-function experiments. A small interfering RNA (siRNA) duplex (siE2F3) targeting E2F3 mRNA was used for RNA interference experiments. All RNA duplexes and oligos were obtained from RiboBio (Guangzhou, China).

Cells $\left(5 \times 10^{5}\right)$ were seeded into 6-well plates (Nest Biotechnology, Hong Kong, China). At $70 \%$ confluency, the cells were subjected to transfection using Lipofectamine 2000 (Invitrogen, Carlsbad, CA, USA) following the manufacturer's instructions (17). The sequences of the RNA duplexes and oligos were as follows: miR-497 mimics, 5'-CAGCAGCACA CUGUGGUUUGU-3'; NC, 5'-UUGUACUACACAAAAGUA CUG-3'; siE2F3, 5'-UAACCUUUGAUUCUCUGAAUCC UCG-3'.

Real-time quantitative RT-PCR. Total RNA was extracted from cells or tissues using an All-in-One microRNA extraction kit (GeneCopoeia, Rockville, MD, USA), according to the manufacturer's protocol. Expression of miR-497 was measured using a one-step qRT-PCR kit (EzOmics SYBR qPCR kit) as previously described (18). An miR-497 stem-loop primer (U6 primer) and EzOmics SYBR qPCR kit were obtained from Biomics Biotechnology Inc. (Jiangsu, China). Real-time PCR was performed with the StepOnePlus Real-Time PCR instrument. The primers were as follows: miR-497, 5'-GTCGTATC CAGTGCAGGGTCCGAGGTATTCGCACTGGATACGAC
ACAAA-3' (stem-loop primer), 5'-CGCCAGCAGCACACTG TGG-3' (sense), and 5'-GTGCAGGGTCCGAGGT-3' (antisense); U6, 5'-GTCCTATCCAGTGCAGGGTCCGAGG TGCACTGGATACGACAAAATATGGAAC-3' (stem-loop primer), 5'-TGCGGGTGCTCGCTTCGCAGC-3' (sense), and 5'-CCAGTGCAGGGTCCGAGGT-3' (antisense). U6 was used as an internal control. All results are representative of three independent assays, and the expression levels of miR-497 were calculated by the $2^{-\Delta \Delta \mathrm{Ct}}$ method.

Cell Counting Kit-8 assay. Cell proliferation was measured using a Cell Counting Kit-8 assay (CCK-8) (Solarbio, Beijing, China). Cells transfected with miR-497 mimics, NC, siRNAs and relative controls were seeded in 96-well plates (Corning, Corning, NY, USA) at $2 \times 10^{3}$ cells/well. At $0,24,48$ and $72 \mathrm{~h}$, $10 \mu \mathrm{l}$ CCK-8 reagent was added to each well, After $2 \mathrm{~h}$ of incubation, the absorbance was measured at $450 \mathrm{~nm}$ as previously described (3). All results are representative of three independent assays.

Colony formation assay. After transfection, 300 cells were seeded into 6-well plates containing $2 \mathrm{ml}$ DMEM supplemented with $10 \% \mathrm{FBS} /$ well and incubated at $37^{\circ} \mathrm{C}$ in a humidified atmosphere with $5 \% \mathrm{CO}_{2}$ for 7-10 days. After staining with a $0.1 \%$ crystal violet solution for $10 \mathrm{~min}$, the numbers of colonies were counted. All results are representative of three independent assays.

Cell migration and invasion assays. At $90 \%$ confluency, a sterile pipette tip was used to make a scratch through each well. Cells were photographed under a microscope at 0 and $24 \mathrm{~h}$ for comparison. We used Transwell invasion assays to evaluate the invasive ability of cells as previously reported (3). An inverted microscope was used to observed cell invasion. The invasion assay was terminated when the cells crossed into the lower well. After the Matrigel was scraped off, the number of cells in the bottom well was counted. Images of cells stained with 4',6-diamidino-2-phenylindole dihydrochloride (DAPI) were obtained. Each treatment was performed in triplicate.

Dual-luciferase reporter assays. Dual-luciferase reporter assays were performed as previously reported (18). Briefly, T24 cells were seeded in 96-well plates and co-transfected with a pMir-Report luciferase vector, pRL-TK Renilla luciferase vector and miR-497 mimics, as previously reported (17). After $48 \mathrm{~h}$, the luciferase activities were determined with the Dual-Luciferase Reporter Assay system (Promega, Madison, WI, USA). Renilla luciferase activity was used as an internal control. After normalization to Renilla luciferase activity, the firefly luciferase activity was analyzed. All results are representative of three independent assays.

E2F3 rescue experiments. The E2F3-coding sequence excluding the 3'-UTR was inserted into a pReceiver vector (GeneCopoeia) to construct the pReceiver-E2F3 vector. The BTCC cells were co-transfected with miR-497 or NC and pReceiverE2F3 or an empty pReceiver vector. BTCC cells were harvested at $48 \mathrm{~h}$ after transfection. Cell proliferation and invasion were determined and E2F3 protein expression was analyzed by western blotting as described above. 

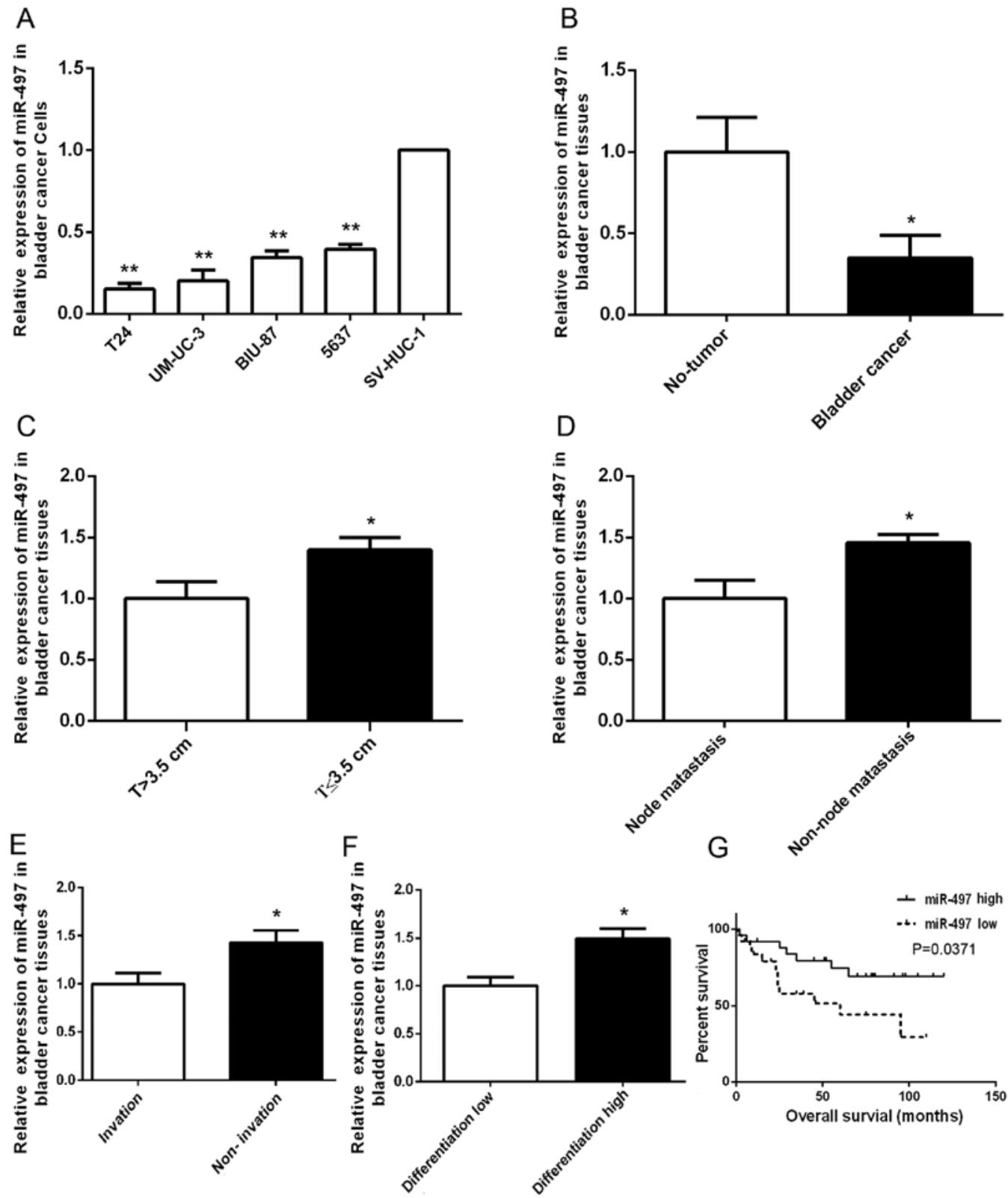

Figure 1. Low expression of miR-497 in human bladder cancer tissues and cell lines, and its correlation with poor prognosis. (A) miR-497 was weakly expressed in four bladder cancer cell lines. (B) miR-497 was weakly expressed in bladder cancer tissues compared with its level in the adjacent tissues in 80 cases. (C) miR- 497 was significantly upregulated in tumor size $(\mathrm{T} \leq 3.5 \mathrm{~cm}$ ) cases compared with cases with $\mathrm{T}>3.5 \mathrm{~cm}$. (D) miR-497 was weakly expressed in patients with lymph node metastasis compared with cases without lymph node metastasis. (E) miR-497 was weakly expressed in patients with local infiltration compared with cases without local infiltration. (F) miR-497 was weakly expressed in patients with lower differentiation compared with patients with relatively higher differentiation. (G) Low expression levels of miR-497 (miR-497 levels in cancer/adjacent tissues $>1$ ) were associated with a poor prognosis compared with high expression levels of miR-497 (miR-497 levels in cancer/adjacent tissues $<1$ ). ${ }^{*} \mathrm{P}<0.05 ;{ }^{* *} \mathrm{P}<0.01$.

Western blot analysis. Proteins were isolated from the cells or tissues by mechanical disruption and a Mammalian Cell Lysis kit (Sigma-Aldrich, St. Louis, MO, USA) according to the manufacturer's instructions. The proteins were separated on $1 \mathrm{~mm}$ NuPage Novex 10\% Bis-Tris gels using a NuPage MOPS SDS Buffer kit (Life Technologies, Carlsbad, CA, USA) followed by electrotransfer to $0.2-\mathrm{mm}$ nitrocellulose membranes (Pall, Port Washington, WI, USA). Non-specific binding sites were blocked with $5 \%$ bovine serum albumin in phosphate-buffered saline (PBS) for $1 \mathrm{~h}$ at room temperature. The membranes were then incubated with a diluted primary antibody (1:1,000; Cell Signaling Technology, Inc., Danvers, MA, USA ) at $4^{\circ} \mathrm{C}$ overnight. After three washes with PBS containing 0.5\% Tween-20, the membranes were incubated with a diluted secondary antibody (GE Healthcare, Buckinghamshire, UK) at room temperature for $2 \mathrm{~h}$. Signals were visualized with enhanced chemiluminescent reagent (Amersham Biosciences, Piscataway, NJ, USA). As a protein loading control, the blots were stripped and stained for GAPDH using an anti-GAPDH antibody (1:2,000; Abcam, Cambridge, MA, USA).

Statistical analysis. Data are expressed as the mean \pm standard deviation (SD). All statistical analyses were performed by one-way analysis of variance (ANOVA) (SPSS 18.0). miRNA target prediction and the associated mRNA pathway analysis 
A
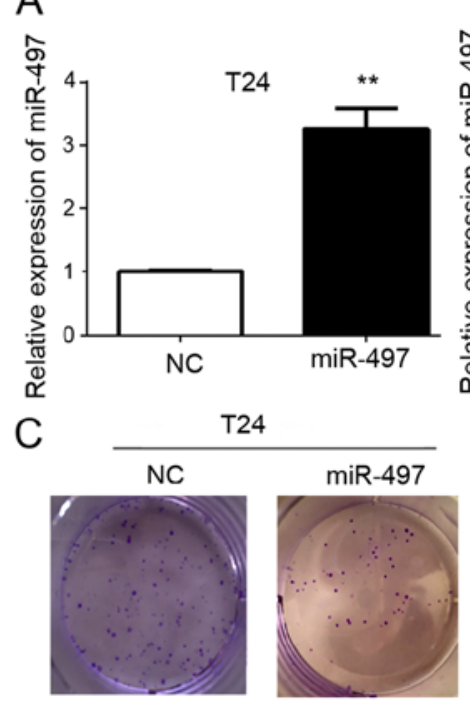

D
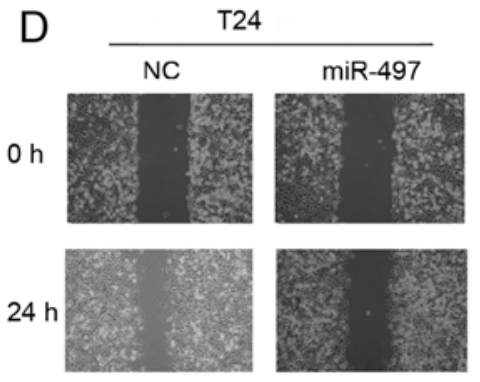

$\mathrm{E}$

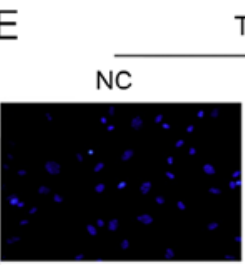

T24
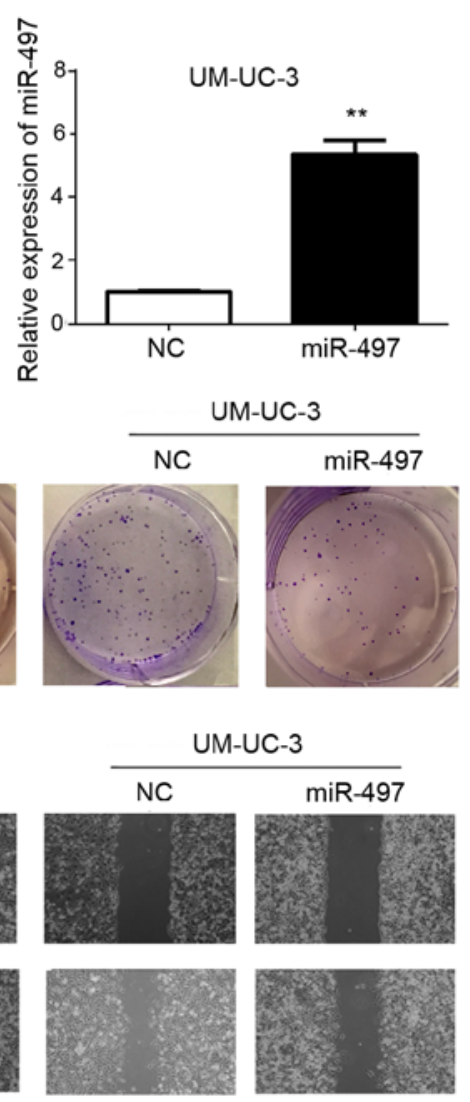

B
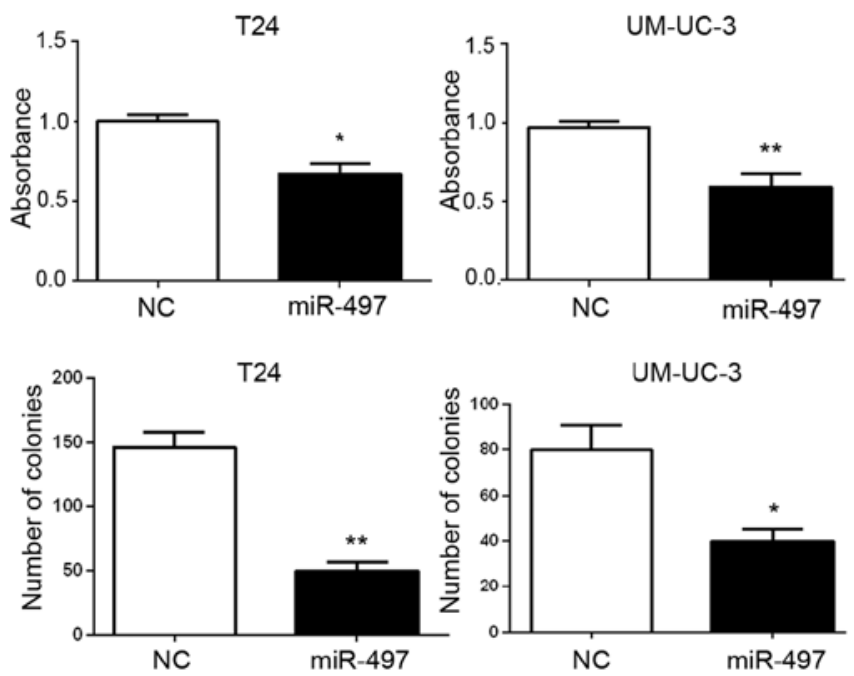
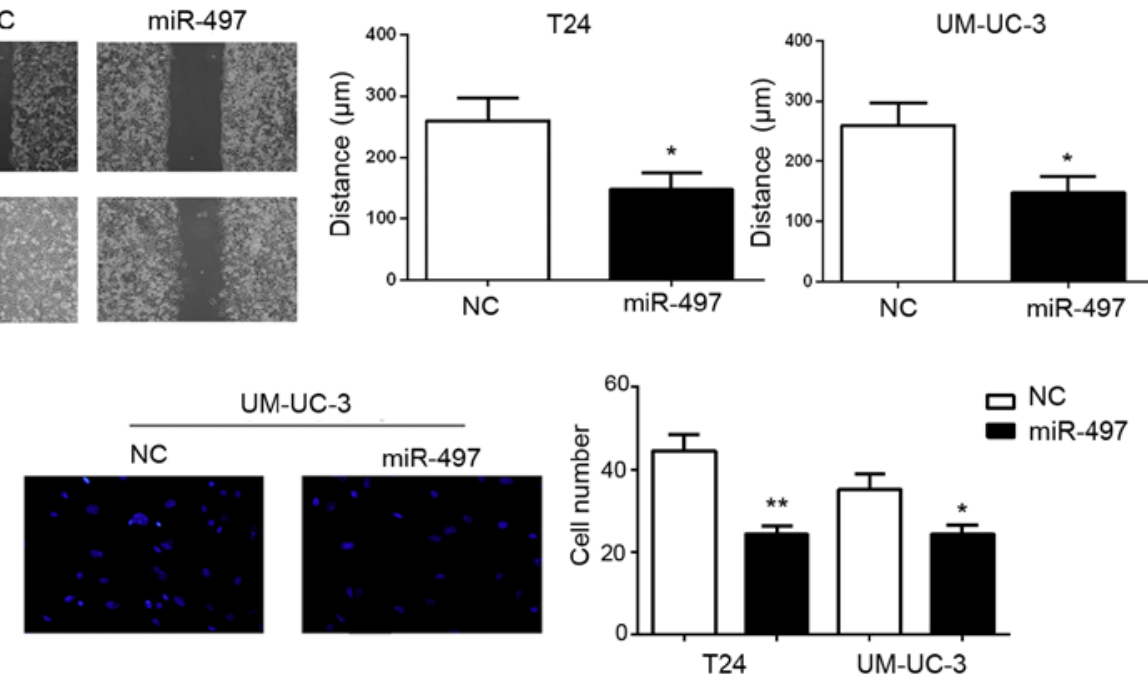

므

- miR-497

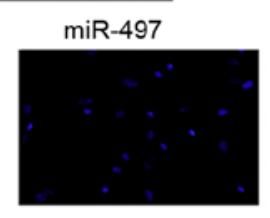

Figure 2. In vitro tumor-suppressing effect of miR-497. (A) T24 and UM-UC-3 cells were transfected with miR-497 or NC, and then miR-497 expression levels were analyzed by quantitative RT-PCR. (B) CCK-8 assays revealed differences in the growth of the indicated cell lines. (C) Colony formation assays of T24 and UMUC-3 cells. (D) Measurement of in vitro cell migration by wound healing assays. Representative images (left) and quantification (right) of the same single areas of the indicated cell lines are shown. (E) Transwell invasion assays of the indicated cell lines. Data are presented as the mean \pm SD of three independent experiments. ${ }^{*} \mathrm{P}<0.05 ;{ }^{* *} \mathrm{P}<0.01$.

were performed using ingenuity pathway analysis and TargetScan. Differences between treatments were assessed using Fisher's least significant difference test. Significant differences were considered at $\mathrm{P}<0.05$.

\section{Results}

miR-497 is downregulated in BTCC cell lines and tissues. We detected the expression of miR-497 in four human BTCC cell lines (T24, 5637, BIU-87 and UM-UC-3) by RT-PCR and found that miR-497 was downregulated in BTCC cell lines T24, 5637, BIU-87 and UM-UC-3 compared with that noted in a normal bladder epithelial cell line, SV-HUC-1 (Fig. 1A). The levels of miR-497 were also detected in 80 human BTCC specimens and adjacent normal tissues by RT-PCR. The results showed that miR-497 was downregulated in the human BTCC specimens compared with that noted in their adjacent normal tissues (Fig. 1B). Moreover, a low level of miR-497 expression was correlated with tumor size (Fig. 1C), node metastasis (Fig. 1D), invasion (Fig. 1E), differentiation (Fig. 1F) and poor prognosis (Fig. 1G).

miR-497 suppresses BTCC progression in vitro. To examine the role of miR-497 in cell proliferation, migration and invasion, T24 and UM-UC-3 cell lines were transfected with miR-497 mimics or NC. Increased expression of miR-497 upon transfection was confirmed by RT-PCR (Fig. 2A). As shown in Fig. 2B, ectopic miR-497 expression suppressed proliferation of the BTCC cells as analyzed by the CCK-8 assay. Consistent with the effects on cell proliferation, the capacity for colony formation by both cell lines was robustly compromised by miR-497 transfection compared with the 
A

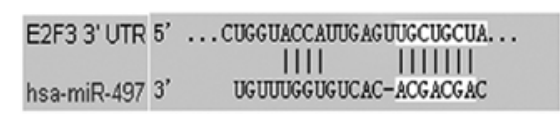

C

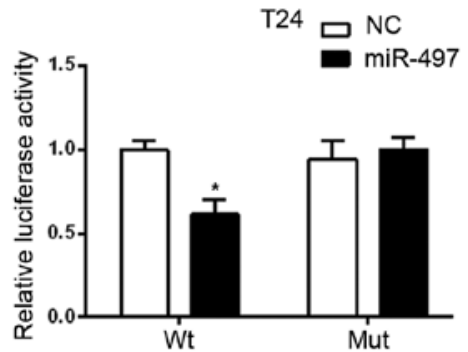

B

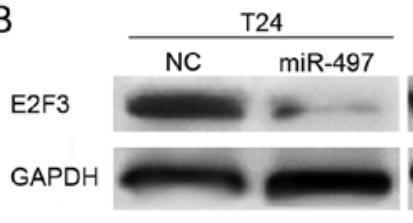

$\mathrm{E}$
D

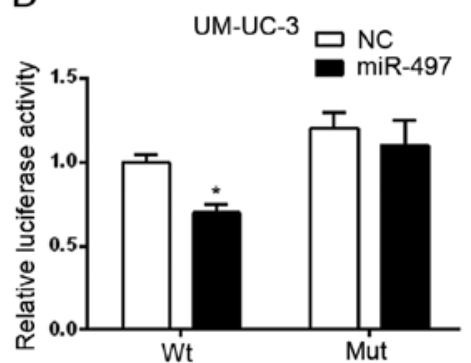

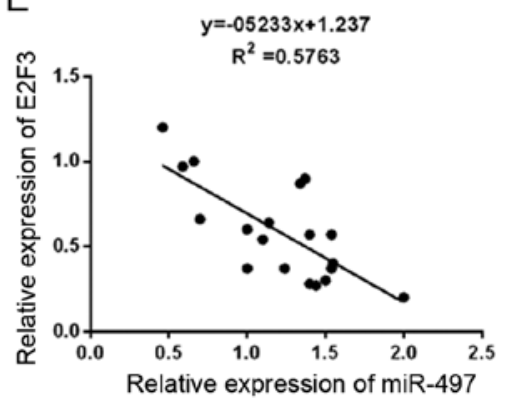

Figure 3. miR-497 directly targets E2F3 in bladder cancer cells. (A) miR-497 directly targets E2F3. (B) Western blotting of E2F3 expression in the indicated cell lines. (C and D) Luciferase activities of the avpGL3-E2F3-3'-UTR reporter in the indicated cells co-transfected with the indicated oligonucleotides. (E) E2F3 protein levels were inversely related with miR-497 levels in human bladder cancer tissues. ${ }^{*} \mathrm{P}<0.05 ;{ }^{* *} \mathrm{P}<0.01$.

corresponding control cells (Fig. 2C). As shown in Fig. 2D, the migratory ability of the miR-497 mimic groups was lower than that of the NC groups. Similar to the effect on cell migration, the capacity for invasion of both cell lines was significantly decreased by miR-497 transfection compared with that noted in the corresponding control cells (Fig. 2E). Taken together, our results revealed that miR-497 inhibited BTCC cell proliferation, migration and invasion in vitro.

miR-497 directly targets E2F3 in BTCC cells. Next, we identified the miR-497 target gene to gain further insight into the molecular mechanisms of miR-497 in BTCC proliferation, migration and invasion. The public database-TargetScan (http://www.targetscan.org) was used to predict the potential target of miR-497. Due to a critically conserved binding site, E2F3 was selected for further examination (Fig. 3A).

To investigate the relationship between miR-497 and E2F3, the protein expression of E2F3 was measured in BTCC cell lines with differential expression of miR-497. E2F3 protein levels in T24 and UM-UC-3 cells were significantly decreased after overexpression of miR-497 mimics (Fig. 3B). To confirm whether E2F3 is a direct target of miR-497, we constructed a dual-luciferase reporter vector with the putative E2F3 3'UTR target site for miR-497, which was downstream of the luciferase gene (pMir-E2F3-Wt), and a deletion mutant of $7 \mathrm{bp}$ in the seed region (pMir-E2F3-Mut). As shown in Fig. 3C and D, luciferase activity assays showed that miR-497 significantly suppressed the activity of Wt, but not Nut reporters in the T24 and UM-UC-3 cells. In addition, the relative expression levels of E2F3 were inversely correlated with the relative expression levels of miR-497 in the BTCC tissues (Fig. 3E). These results strongly demonstrated the specificity of miR-497 to target E2F3.

siE2F3 inhibits the proliferation, migration and invasion of BTCC cells. As shown in Fig. 4A and B, compared with NC, siE2F3 decreased the expression of E2F3 at both the mRNA and protein levels. To reveal the biological function of E2F3, siE2F3 was stably transfected into the T24 and UM-UC-3 cells. As a result, we found that siE2F3 could reproduce an effect on cell proliferation, migration and invasion similar to that of miR-497 (Fig. 4C-F).

Restoration of E2F3 partially rescues miR-497-induced inhibition of the proliferation, migration and invasion of BTCC cells. To further explore whether overexpression of E2F3 could rescue the suppression of cell proliferation, migration and invasion induced by miR-497, we inserted the E2F3-coding sequence excluding the 3-UTR into the pReceiver vector, and found that $\mathrm{E} 2 \mathrm{~F} 3$ expression levels were enhanced by transfection in the T24 cells (Fig. 5A). Cell proliferation, migration and invasion were evaluated in cells co-transfected with both miR-497 mimics or NC and pReceiver-E2F3 or an empty pReceiver vector. Our results indicated that overexpression of E2F3 could partially rescue miR-497-induced inhibition of cell proliferation, migration and invasion (Fig. 5B-F).

\section{Discussion}

Recent studies have demonstrated the potential of miRNAs as promising prognostic biomarkers $(2,3)$. Targeting miRNAs is an effective approach for the treatment of advanced cancers (2-4). Moreover, aberrant expression of miRNAs has been identified in bladder cancer, which plays crucial roles in tumorigenesis and cancer progression. miR-497 is a recently discovered miRNA that is downregulated in bladder, gastric and breast cancers (19-24). In the present study, we found that miR-497 was markedly downregulated in the BTCC cell lines. Our results indicate that low expression of miR-497 is significantly associated with BTCC progression.

We also analyzed the relative expression of miR-497 in BTCC tissues. miR-497 expression was decreased in cancer tissues, and the low expression of miR-497 was significantly associated with tumor size, node metastasis, invasion, differentiation, as well as a poor prognosis. These results imply that miR-497 expression may function as an independent prognosis factor that is significantly associated with the overall survival rates of BTCC patients and BTCC progression. Our results 
A

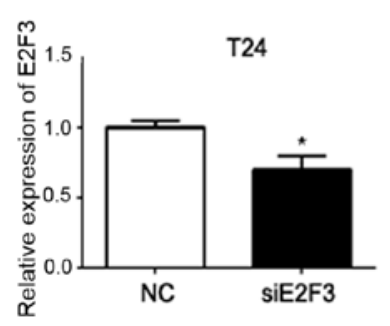

C

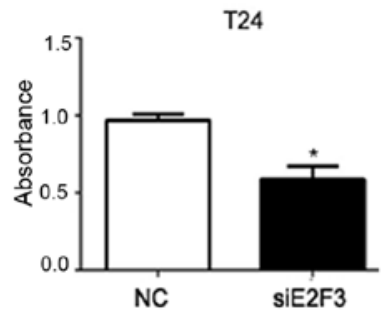

E

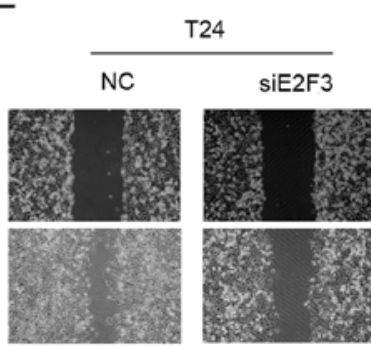

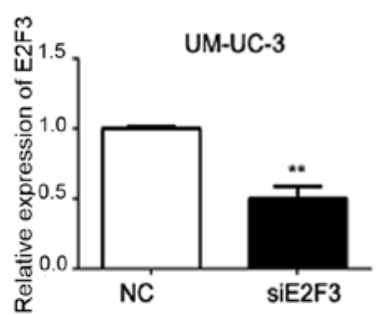

UM-UC-3

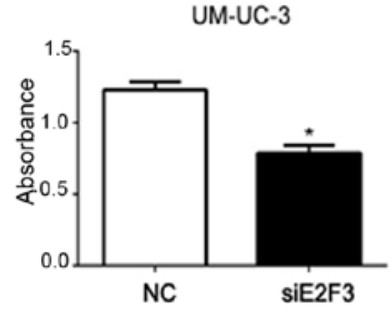

B

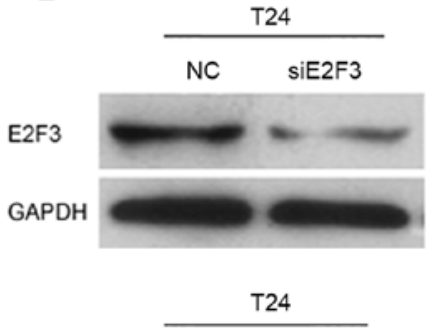

$\mathrm{D}$
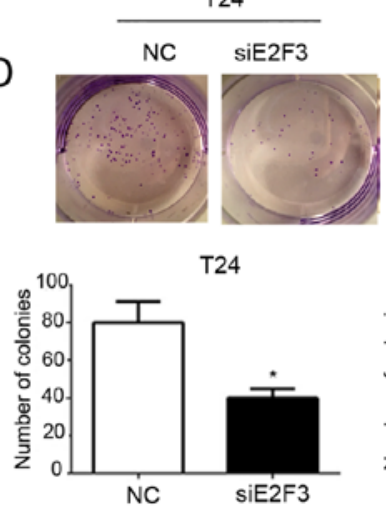

T24
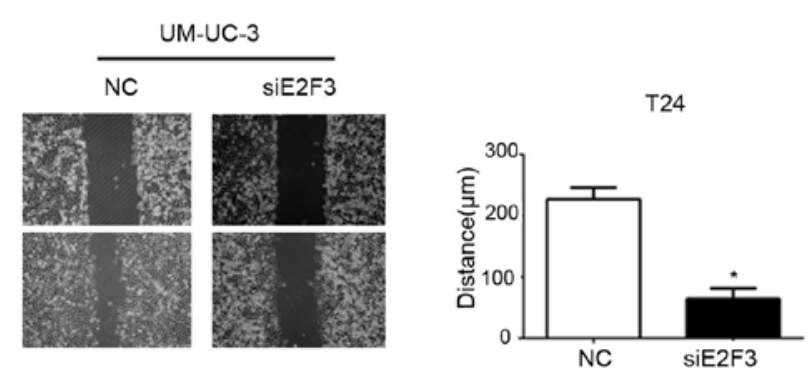

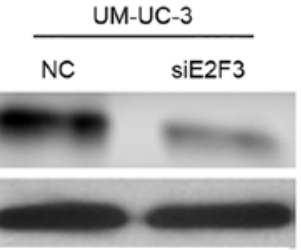

UM-UC-3

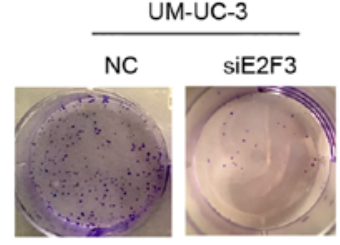

UM-UC-3

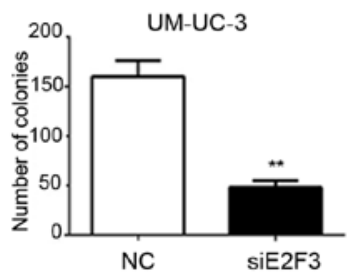

UM-UC-3

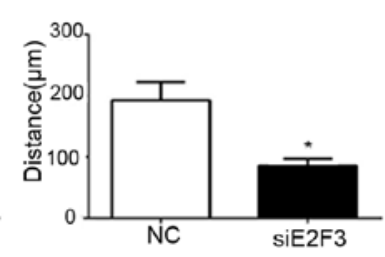

$\mathrm{F}$
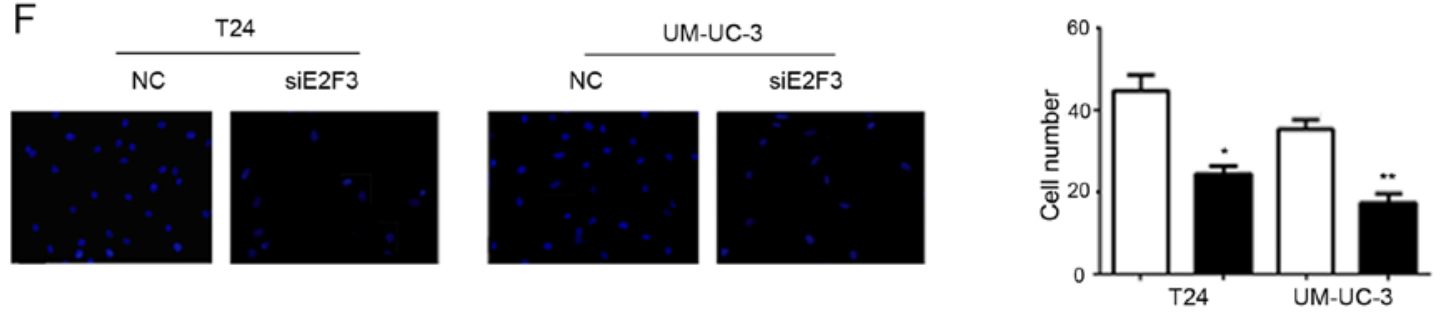

$\square \mathrm{NC}$

- $\operatorname{siE} 2 \mathrm{~F} 3$

Figure 4. Knockdown of E2F3 phenocopies the effect of miR-497. T24 and UM-UC-3 cells were tranfected with siE2F3 or NC. (A and B) Knockdown of E2F3 reduced the expression of E2F3 at mRNA and protein levels in both cell lines. (C) CCK-8 assays revealed differences in the growth rate of the indicated cell lines. (D) Colony formation assays of T24 and UM-UC-3 cells. (E) Measurement of in vitro cell migration by wound healing assays. Representative images (left) and quantification (right) of the same areas of the indicated cell lines are shown. (F) Transwell invasion assays of the indicated cell lines. Data are presented as the mean \pm SD of three independent experiments. ${ }^{*} \mathrm{P}<0.05 ;{ }^{* *} \mathrm{P}<0.01$.

were consistent with a previous study by Zhao et al who showed that downregulation of miR-497 is associated with a poor prognosis in renal cancer (25). Further studies demonstrated that upregulation of miR-497 significantly decreased cell proliferation, the colony formation rate, wound healing and migration rates, indicating that miR-497 inhibits the proliferation, migration and invasion of BTCC cells.

Next, we explored the molecular mechanism by which miR-497 acts as a suppressor in BTCC progression. Luciferase reporter assays and western blotting were employed to confirm that E2F3 is direct target of miR-497 in BTCC cells. E2F3, which is known as a transcription factor, has a central role in linking cell cycle proteins, such as cyclins, cyclin-dependent kinases and $\mathrm{pRB}$, to the expression of genes involved in cell growth and survival (26-29).
E2F3 gene amplification and protein overexpression have been extensively studied in bladder cancer (10). High levels of E2F3 expression have been observed in approximately one-third of BTCC with E2F3 overexpression increasing with tumor grade and stage (10). Hence, the mechanism of E2F3 upregulation, except for gene RNA inference, recent reports showed several miRNAs (miR-141, miR-199a-5p, miR-125 and miR-200c) may be involved (30-34). In the present study, western blot analysis and RT-PCR demonstrated downregulation of E2F3 by miR-497, which inhibited the cell proliferation, migration and invasion of BTCC cells. To test whether miR-497 inhibits proliferation, migration and invasion of BTCC cells through targeting E2F3, the expression of E2F3 was knocked down using specific siRNAs. siE2F3 could emulate the effects of miR-497 overexpression on cell 
A

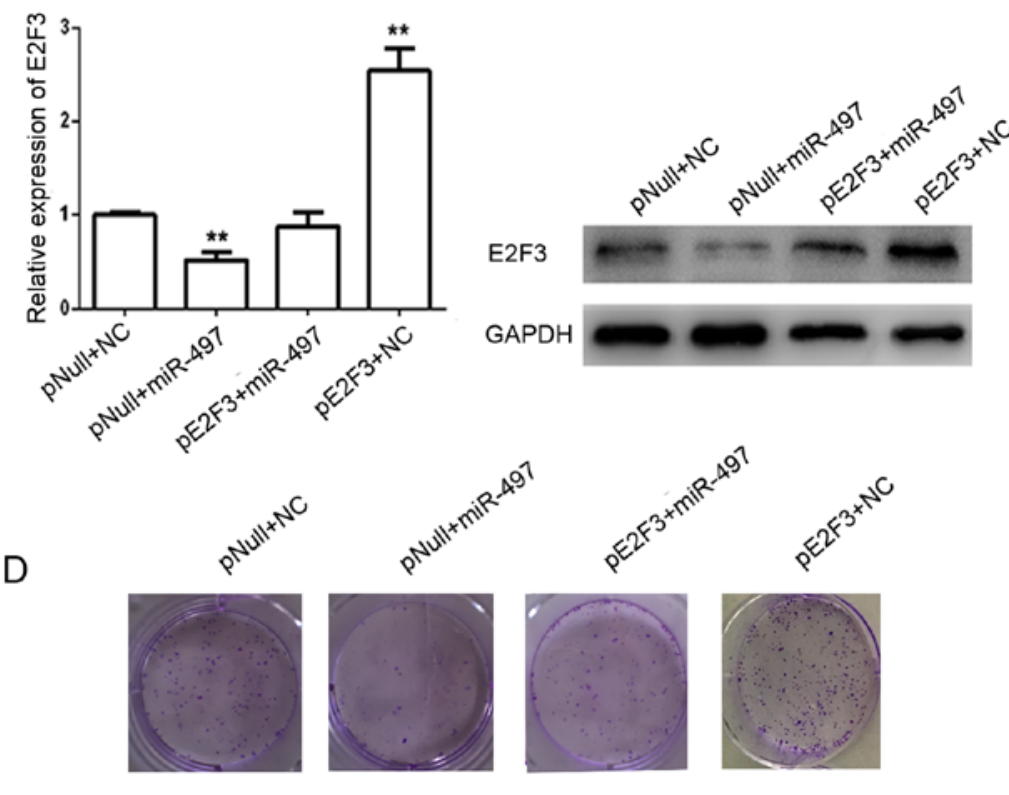

C

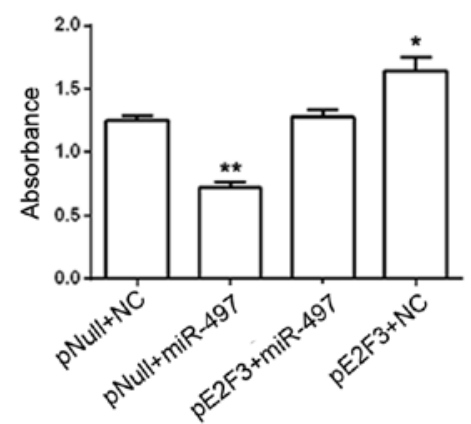

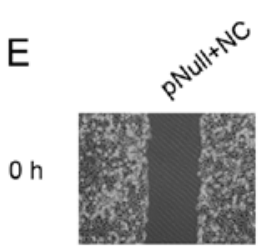

$24 \mathrm{~h}$

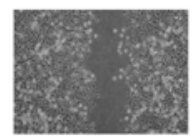

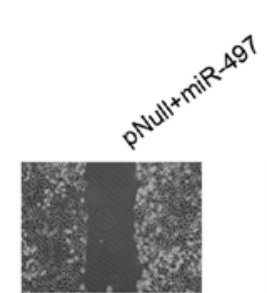
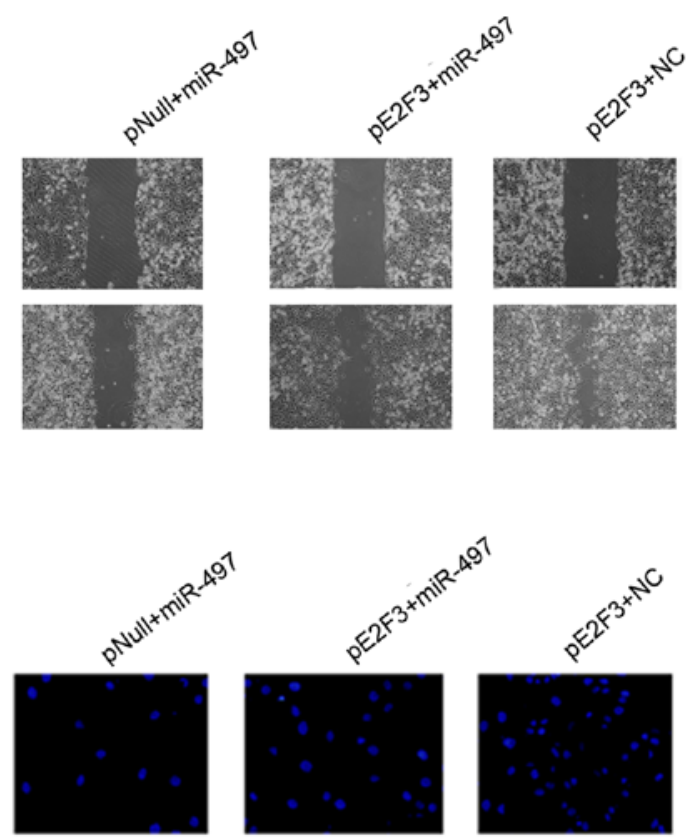

$\mathrm{F}$

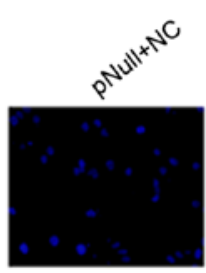

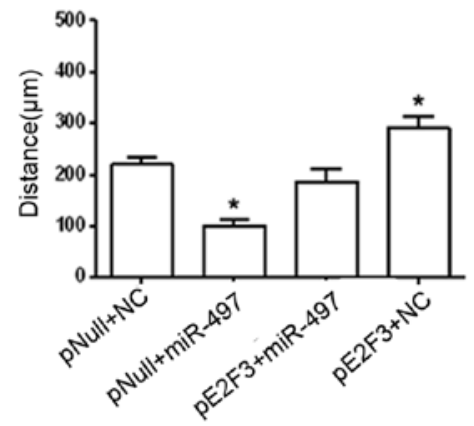

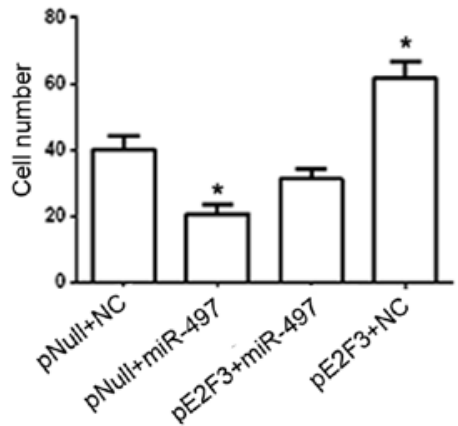

Figure 5. Forced expression of E2F3 rescues miR-497-induced inhibition of cell proliferation, migration and invasion. (A) T24 cells were co-transfected with both miR-497 or NC and pReceiver-E2F3 or an empty pReceiver vector. The expression of E2F3 or GAPDH was detected by real-time PCR. (B) T24 cells were co-transfected with both miR-497 or NC and pReceiver-E2F3 or an empty pReceiver vector. The expression of E2F3 or GAPDH was detected by western blot analysis. (C) CCK-8 assays revealed differences in the growth of the indicated cell lines. (D) Colony formation assays of T24 cells. (E) Measurement of in vitro cell migration by wound healing assays. Representative images (left) and quantification (right) of the same area of the indicated cell lines. (F) Transwell invasion assays of the indicated cell lines. Representative images (left) and quantification (right) of the same area of the indicated cell lines. Data are presented as the mean $\pm \mathrm{SD}$ of three independent experiments. ${ }^{*} \mathrm{P}<0.05 ;{ }^{* *} \mathrm{P}<0.01$.

growth, migration and invasion. All these results suggest that miR-497 may act as a suppressor of BTCC cells by inhibiting the expression of E2F3. Therefore, we proposed that downregulation of miR-497 may enhance the proliferation and invasion of BTCC cells and subsequently facilitate the development of BTCC through upregulating the expression of E2F3. The rescue effect of E2F3 expression partly reversed the inhibition of cell proliferation, migration and invasion induced by miR-497. In the present study, we demonstrated the direct role of E2F3 in the proliferation, migration and invasion of BTCC cells, which were regulated by miR-497. The specific mechanism will be revealed in a future study.

In conclusion, our data demonstrated altered expression of miR-497 in human BTCC cell lines and BTCC tissues, and 
that low levels of miR-497 correlate with poor prognoses in patients. Our data also showed that miR-497 suppresses the proliferation and invasion of BTCC cells, possibly through modulating the target gene E2F3. These findings suggest that miR-497 may be valuable biomarker for BTCC progression, and the miR-497-E2F3 axis may be a novel therapeutic target for BTCC.

\section{Acknowledgements}

The present study was supported by research grants from the National Natural Science Foundation of China (no. 81372723), and the Key Urology Laboratory Foundation of Shenyang City of China (F13-293-1-00).

\section{References}

1. Siegel R, Ma J, Zou Z and Jemal A: Cancer statistics, 2014. CA Cancer J Clin 64: 9-29, 2014

2. Su Z, Yang Z, Xu Y, Chen Y and Yu Q: MicroRNAs in apoptosis, autophagy and necroptosis. Oncotarget 6: 8474-8490, 2015.

3. Liu L, Qiu M, Tan G, Liang Z, Qin Y, Chen L, Chen H and Liu J: miR-200c inhibits invasion, migration and proliferation of bladder cancer cells through down-regulation of BMI-1 and E2F3. J Transl Med 12: 305, 2014.

4. Luo Q, Li X, Gao Y, Long Y, Chen L, Huang Y and Fang L: MiRNA-497 regulates cell growth and invasion by targeting cyclin E1 in breast cancer. Cancer Cell Int 13: 95, 2013.

5. Wang W, Ren F, Wu Q, Jiang D, Li H and Shi H: MicroRNA-497 suppresses angiogenesis by targeting vascular endothelial growth factor A through the PI3K/AKT and MAPK/ERK pathways in ovarian cancer. Oncol Rep 32: 2127-2133, 2014.

6. Li W, Jin X, Deng X, Zhang G, Zhang B and Ma L: The putative tumor suppressor microRNA-497 modulates gastric cancer cell proliferation and invasion by repressing eIF4E. Biochem Biophys Res Commun 449: 235-240, 2014.

7. Itesako T, Seki N, Yoshino H, Chiyomaru T, Yamasaki T, Hidaka H, Yonezawa T, Nohata N, Kinoshita T, Nakagawa M, et al: The microRNA expression signature of bladder cancer by deep sequencing: The functional significance of the miR-195/497 cluster. PLoS One 9: e84311, 2014.

8. Vimala K, Sundarraj S, Sujitha MV and Kannan S: Curtailing overexpression of E2F3 in breast cancer using siRNA (E2F3)-based gene silencing. Arch Med Res 43: 415-422, 2012.

9. Hurst CD, Tomlinson DC, Williams SV, Platt FM and Knowles MA: Inactivation of the Rb pathway and overexpression of both isoforms of E2F3 are obligate events in bladder tumours with 6p22 amplification. Oncogene 27: 2716-2727, 2008.

10. Olsson AY, Feber A, Edwards S, Te Poele R, Giddings I, Merson S and Cooper CS: Role of E2F3 expression in modulating cellular proliferation rate in human bladder and prostate cancer cells. Oncogene 26: 1028-1037, 2007.

11. Shen H, Morrison CD, Zhang J, Underwood W III, Yang N, Frangou C, Eng K, Head K, Bollag RJ, Kavuri SK, et al: 6p22.3 amplification as a biomarker and potential therapeutic target of advanced stage bladder cancer. Oncotarget 4: 2124-2134, 2013.

12. Huang L, Luo J, Cai Q, Pan Q, Zeng H, Guo Z, Dong W, Huang J and Lin T: MicroRNA-125b suppresses the development of bladder cancer by targeting E2F3. Int J Cancer 128: 1758-1769, 2011.

13. Oeggerli M, Tomovska S, Schraml P, Calvano-Forte D, Schafroth S, Simon R, Gasser T, Mihatsch MJ and Sauter G: E2F3 amplification and overexpression is associated with invasive tumor growth and rapid tumor cell proliferation in urinary bladder cancer. Oncogene 23: 5616-5623, 2004

14. Welch C, Chen Y and Stallings RL: MicroRNA-34a functions as a potential tumor suppressor by inducing apoptosis in neuroblastoma cells. Oncogene 26: 5017-5022, 2007.

15. Sylvestre Y, De Guire V, Querido E, Mukhopadhyay UK, Bourdeau V, Major F, Ferbeyre G and Chartrand P: An E2F/miR-20a autoregulatory feedback loop. J Biol Chem 282: 2135-2143, 2007
16. Su J, Wang Q, Liu $Y$ and Zhong M: miR-217 inhibits invasion of hepatocellular carcinoma cells through direct suppression of E2F3. Mol Cell Biochem 392: 289-296, 2014.

17. Xu Y, Zhao F, Wang Z, Song Y, Luo Y, Zhang X, Jiang L, Sun Z, Miao Z and Xu H: MicroRNA-335 acts as a metastasis suppressor in gastric cancer by targeting Bcl-w and specificity protein 1. Oncogene 31: 1398-1407, 2012.

18. Iseki H, Takagi M, Kuroda Y, Katsuda K, Mikami O, Tsunemitsu $\mathrm{H}$ and Yamakawa M: Application of a SYBR ${ }^{\circledR}$ Green one step real-time RT-PCR assay to detect type 1 porcine reproductive and respiratory syndrome virus. J Vet Med Sci 76: 1411-1413, 2014.

19. Xu J, Wang T, Cao Z, Huang H, Li J, Liu W, Liu S, You L, Zhou L, Zhang T, et al: MiR-497 downregulation contributes to the malignancy of pancreatic cancer and associates with a poor prognosis. Oncotarget 5: 6983-6993, 2014.

20. Zhao WY, Wang Y, An ZJ, Shi CG, Zhu GA, Wang B, Lu MY, Pan CK and Chen P: Downregulation of miR-497 promotes tumor growth and angiogenesis by targeting HDGF in non-small cell lung cancer. Biochem Biophys Res Commun 435: 466-471, 2013.

21. Wang L, Li B, Li L and Wang T: MicroRNA-497 suppresses proliferation and induces apoptosis in prostate cancer cells. Asian Pac J Cancer Prev 14: 3499-3502, 2013.

22. Shen L, Li J, Xu L, Ma J, Li H, Xiao X, Zhao J and Fang L: miR-497 induces apoptosis of breast cancer cells by targeting Bcl-w. Exp Ther Med 3: 475-480, 2012.

23. Guo ST, Jiang CC, Wang GP, Li YP, Wang CY, Guo XY, Yang RH, Feng Y, Wang FH, Tseng HY, et al: MicroRNA-497 targets insulin-like growth factor 1 receptor and has a tumour suppressive role in human colorectal cancer. Oncogene 32: 1910-1920, 2013

24. Flavin RJ, Smyth PC, Laios A, O'Toole SA, Barrett C, Finn SP, Russell S, Ring M, Denning KM, Li J, et al: Potentially important microRNA cluster on chromosome $17 \mathrm{p} 13.1$ in primary peritoneal carcinoma. Mod Pathol 22: 197-205, 2009.

25. Zhao X, Zhao Z, Xu W, Hou J and Du X: Down-regulation of miR-497 is associated with poor prognosis in renal cancer. Int $\mathrm{J}$ Clin Exp Pathol 8: 758-764, 2015.

26. Chang S, Gao L, Yang Y, Tong D, Guo B, Liu L, Li Z, Song T and Huang C: miR-145 mediates the antiproliferative and gene regulatory effects of vitamin D3 by directly targeting E2F3 in gastric cancer cells. Oncotarget 6: 7675-7685, 2015.

27. Kanai D, Ueda A, Akagi T, Yokota $T$ and Koide H: Oct $3 / 4$ directly regulates expression of E2F3a in mouse embryonic stem cells. Biochem Biophys Res Commun 459: 374-378, 2015.

28. Chen PH, Shih CM, Chang WC, Cheng CH, Lin CW, Ho KH, Su PC and Chen KC: MicroRNA-302b-inhibited E2F3 transcription factor is related to all trans retinoic acid-induced glioma cell apoptosis. J Neurochem 131: 731-742, 2014.

29. An Q, Wang Y, An R, Li Y, Yao T, Zhai B and Sun X: Association of E2F3 expression with clinicopathological features of Wilms' tumors. J Pediatr Surg 48: 2187-2193, 2013.

30. Cooper CS, Nicholson AG, Foster C, Dodson A, Edwards S, Fletcher A, Roe T, Clark J, Joshi A, Norman A, et al: Nuclear overexpression of the E2F3 transcription factor in human lung cancer. Lung Cancer 54: 155-162, 2006.

31. Tao T, Liu D, Liu C, Xu B, Chen S, Yin Y, Ang L, Huang Y, Zhang $X$ and Chen M: Autoregulatory feedback loop of $\mathrm{EZH} 2 / \mathrm{miR}-200 \mathrm{c} / \mathrm{E} 2 \mathrm{~F} 3$ as a driving force for prostate cancer development. Biochim Biophys Acta 1839: 858-865, 2014.

32. Song C, Wu G, Xiang A, Zhang Q, Li W, Yang G, Shi X, Sun S and $\mathrm{Li} \mathrm{X}$ : Over-expression of miR-125a-5p inhibits proliferation in $\mathrm{C} 2 \mathrm{C} 12$ myoblasts by targeting E2F3. Acta Biochim Biophys Sin 47: 244-249, 2015.

33. Xue J, Niu YF, Huang J, Peng G, Wang LX, Yang YH and Li YQ: miR-141 suppresses the growth and metastasis of HCC cells by targeting E2F3. Tumour Biol 35: 12103-12107, 2014.

34. Lee JM, Heo MJ, Lee CG, Yang YM and Kim SG: Increase of miR-199a-5p by protoporphyrin IX, a photocatalyzer, directly inhibits E2F3, sensitizing mesenchymal tumor cells to anticancer agents. Oncotarget 6: 3918-3931, 2015. 